\title{
Journal of Experimental Neuroscience
}

\section{Altered Postnatal Cell Proliferation in Brains of Mouse Pups Prenatally Exposed to IgG from Mothers of Children with Autistic Disorder}

\author{
Shilpa D. Kadam ${ }^{1,3}$, Beth M. French ${ }^{5}$, S-T. Kim ${ }^{1}$, Christy M. Morris-Berry ${ }^{5}$, Andrew W. Zimmerman², \\ Mary E. Blue ${ }^{1,3,4}$ and Harvey S. Singer ${ }^{3,5}$ \\ ${ }^{1}$ Neuroscience Laboratory, Hugo W Moser Research Institute; ${ }^{2}$ Department of Neurology and Developmental Medicine at Kennedy Krieger; \\ ${ }^{3}$ Departments of Neurology, ${ }^{4}$ Neuroscience, and ${ }^{5}$ Pediatrics, Johns Hopkins University School of Medicine, Baltimore, MD, USA.
}

\begin{abstract}
Auto antibodies found in the mothers of children with autistic disorder (MCAD) when passively transferred to pregnant mice cause behavioral alterations in juvenile and adult offspring. The goal of this study was to identify whether intraperitoneal injection of MCAD-IgG during gestation affected postnatal cell proliferation and survival in P7 offspring. Pooled MCAD-IgG or IgG from mothers of unaffected children (MUC) or phosphate-buffered saline was injected daily into C57BL/J6 pregnant dams (gestational days E13-E18). MCAD-IgG exposure significantly increased cell proliferation in the subventricular and subgranular zones. In contrast, BrdU-labeled cells on P1 and surviving until P7 (P1-generated cells) showed reduced cell densities in layers 2-4 of frontal and parietal cortices of MCAD mice compared to those in MUC and PBS-injected mice. In conclusion, significant increases in cell proliferation at P7 and reduced densities of P1-generated cells distinguish in utero exposure to MCAD compared to MUC and PBS.
\end{abstract}

KEYWORDS: autism, maternal autoantibodies, neurogenesis, in utero, postnatal, BrdU

CITATION: Kadam et al. Altered Postnatal Cell Proliferation in Brains of Mouse Pups Prenatally Exposed to IgG from Mothers of Children with Autistic Disorder. Journal of Experimental Neuroscience 2013:7 93-99 doi:10.4137/JEN.S12979.

TYPE: Original Research

FUNDING: Author(s) disclose no funding sources.

COMPETING INTERESTS: Author(s) disclose no potential conflicts of interest.

COPYRIGHT: $\odot$ the authors, publisher and licensee Libertas Academica Limited. This is an open-access article distributed under the terms of the Creative Commons CC-BY-NC 3.0 License.

CORRESPONDENCE: kadam@kennedykrieger.org

\section{Introduction}

Autism spectrum disorders (ASDs) are characterized by impairments in sociability, verbal, and non-verbal communication and the presence of repetitive, stereotypical behaviors and restricted interests. ${ }^{1}$ A variety of genetic, biochemical, and environmental factors have been proposed to have roles in causing autism, but definitive evidence is lacking. ${ }^{2}$ The role of the maternal immune system in fetal brain development is an area of active research. ${ }^{3-6}$ In humans, maternal antibodies are detected in fetal circulation as early as 13 weeks of gestation, and their concentration increases to approximately 50\% of maternal levels by 30 weeks of gestation. ${ }^{7}$ It has been estimated that approximately $12 \%$ of mothers of children with autistic disorder (MCAD) have antibodies that are reactive to fetal brain proteins at 37 and $73 \mathrm{kDa} .{ }^{4,8}$

Studies reporting the effects of maternal antibodies on monkey and rodent offspring behavior following gestational exposure indicate that antibodies are a plausible causative factor in a subset of ASD cases. ${ }^{9-11}$ In a pregnant mouse model, offspring of dams receiving in utero MCAD$\mathrm{IgG}$ exposure showed changes in activity, anxiety, startle reflexes, and sociability. ${ }^{10}$ The goal of this current study was to investigate cell proliferation in the postnatal brains of gestationally exposed mice pups in the same pregnant mouse model.

\section{Materials and Methods}

All research was conducted according to a protocols approved by the Johns Hopkins University School of Medicine Animal Care and Use Committee (IACUC).

Animals. Pregnant C57BL/J6 ( $\mathrm{n}=7)$ female mice were purchased from Jackson Laboratory (Bar Harbor, ME, USA) and maintained in the certified animal facility of the Johns Hopkins Broadway Research Building. All investigations 
in the offspring were performed blinded to treatment and without regard to biological sex, the distribution of which could not be controlled for each treatment group with the gestational treatment paradigm used in this study.

Isolation of IgG from human sera. IgG was isolated from pooled serum samples of $63 \mathrm{MCAD}$ and 63 mothers of unaffected children (IgG-controls) who were participants in a previously published study. ${ }^{10}$ Autistic disorder was diagnosed in children based on the presence of abnormalities in social and communication development, marked repetitive behavior, and limited imagination using the Diagnostic and Statistical Manual for Mental Disorders-IV (DSM-IV) and Autism Diagnostic Observation Schedule-Generic or Childhood Autism Rating Scale (CARS). All were judged clinically to have moderate to severe adaptive deficits or cognitive deficits (IQ $<70)$ by formal testing. Children with diagnoses of Asperger syndrome, Pervasive Developmental Disorder-Not Otherwise Specified (PDD-NOS), and those with established genetic or metabolic causes of autism were excluded. An equal quantity of previously frozen, never re-frozen, serum from each subject in the cohort, $225 \mu \mathrm{L}$, was pooled, filtered, and the $\mathrm{IgG}$ isolated by passage over a Protein A cartridge (Sigma, St Louis, MO, USA) according to the manufacturer's protocol. IgG was eluted from the column using acid (elution buffer) and then immediately passed over a desalting cartridge to set the eluate to physiological $\mathrm{pH}$ in phosphate-buffered saline (PBS). Purified $\operatorname{IgG}$ was then filtered $(0.22 \mu \mathrm{m})$ and maintained at $4^{\circ} \mathrm{C}$. The pooled $\operatorname{IgG}$ concentration was determined by rate nephelometry using the Beckman IMMAGE 800 nephelometer (Brea, CA, USA) in the Johns Hopkins Hospital Clinical laboratory; MCAD 279 mg/dL, IgGcontrol $245 \mathrm{mg} / \mathrm{dL}$.

Intraperitoneal injections and treatment groups. Seven pregnant dams received daily intraperitoneal injections of $0.5 \mathrm{~mL}$ of purified IgG from MCAD, IgG-controls, or PBS on gestational days E13 through E18 (3PBS, 2 MUC and 2 MCAD). A total of 48 pups were born to the 7 dams (17 PBS, 18 MUC and 13 MCAD). Three pups $(n=3 / 48)$ died before the P7 harvest (i.e., $\mathrm{n}=45,1$ pup from each treatment group). Bromodeoxyuridine (BrdU), $30 \mathrm{mg} / \mathrm{kg}$ at a concentration $30 \mathrm{mg} / \mathrm{mL}$, was injected into newborn mice on postnatal day 1 (P1; group 1; day of birth = P0), except for 2 mice from each litter, which were injected with BrdU 2 h before euthanasia on postnatal day 7 (P7; group 2; Fig. 1A). All pups were euthanized on P7. In this study, group 1 (ie, cell survival/maturation) contained a total of 35 pups with sample sizes of $12 \mathrm{PBS}, 14 \mathrm{MUC}$, and $9 \mathrm{MCAD}$ pups each. Group 2 (ie, cell proliferation) contained 10 pups with sample sizes of $4 \mathrm{PBS}, 3 \mathrm{MUC}$, and $3 \mathrm{MCAD}$ pups each (Suppl. Fig. 1 - see group 1 vs group 2).

Brain fixation and sectioning. All animals were anesthetized with ether on P7. Mice were then transcardially perfused with ice-cold PBS, $\mathrm{pH} 7.4$ and brains were removed rapidly, post-fixed in methacarn fixative $(60 \%$ methanol, 30\% chloroform, and 10\% glacial acetic acid), and paraffin-embedded. Brains were coronally sectioned at $25 \mu \mathrm{m}$ on a rotary microtome and 3 consecutive sections mounted per slide. One slide from each experimental group was Nisslstained ( 3 sections each). Serial sections $(n=2)$ from each brain were then analyzed using MCID 7.0 Elite (Inter Focus Imaging Ltd, Cambridge, UK) to determine the thickness of the cortex in bilateral hemispheres of the brain (sampling sites $=4$ per brain) at the locations indicated in Figure 1B [atlas coordinates frontal $\sim$ bregma $0.70-0.90 \mathrm{~mm}$ and parietal $\sim$ bregma $-1.70-1.90 \mathrm{~mm}]{ }^{12}$ An adjacent series of consecutively mounted sections were stained for the following: 1 . BrdU + Hoechst (groups 1 and 2); 2. BrdU + Iba-1 + Hoechst (group 1 only; Table 2); and 3. Aldh1 L1 + NeuN + Hoechst (group 1 only). Every brain from each stained series was quantitated in 2 of the 3 sections per slide in bilateral hemispheres at all the ROIs listed in Figure 2.

Immunohistochemistry (IHC). Each deparaffinized slide was subjected to heat-induced antigen retrieval with $10 \mathrm{mM}$ sodium citrate, $\mathrm{pH}$ 6.0, with $0.5 \%$ Triton-X 100, and then incubated in blocking solution (10\% normal serum, $0.5 \%$ Triton X-100 in PBS) at room temperature for $1 \mathrm{~h}$ before proceeding with IHC.

Bromodeoxyuridine(BrdU)staining. Sectionswereincubated with $\mathrm{HCl}$ at $37^{\circ} \mathrm{C}$ for 20 min to denature the DNA, and then neutralized with borate buffer. PBS washes were followed by incubation with the primary antibody Anti-BrdU (Roche; $1: 200$ with $3 \%$ normal goat serum and $0.5 \%$ TritonX-100 in PBS) overnight at $4{ }^{\circ} \mathrm{C}$ in the dark followed by incubation with secondary antibody G $\alpha$ M Alexa 488 (1:400 with 3\% normal goat serum, $0.5 \%$ Triton in PBS; Invitrogen, Carlsbad, CA, USA) at room temperature for $2 \mathrm{~h}$ in the dark. Sections were counterstained with Hoechst diluted 1:2000 in PBS at room temperature; MOWIOL mounting medium (Calbiochem, La Jolla, CA, USA) was applied to each slide and specimens were stored at $4^{\circ} \mathrm{C}$ until imaging.

Iba-1/BrdU staining. Adjacent series of sections on consecutive slides wereincubated with Anti-Iba-1 (1:250 with 3\% normal donkey serum, 0.5\% TritonX-100 in PBS; Wako Pure Chemical Industries, Ltd, Osaka, Japan) followed by BrdU double-labeling (see above).

NeuN/ALDH1L1 staining. Astrocyte-specific marker Aldh1 L1 (Suppl. Fig. 4), ${ }^{13}$ was used to label gray matter and white matter astrocytes and did not label the radial glial stemcells in the neurogenic niches that were GFAPpositive (Suppl. Fig. 4A). Aldh1 L1-labeled sections from a consecutive slide were co-labeled with NeuN [Rabbit antiALDH1L1 (Abcam; 1:1000 with 3\% normal goat serum, $0.5 \%$ Triton X-100 in PBS; Cambridge, MA, USA) and mouse anti-NeuN].

Imaging and quantification using apotome fluorescent microscope, image $\mathbf{J}$ and neurolucida. Axiovision software (Carl Zeiss, Jena, Germany) was used to digitally capture all images. Z-stack images of fluorescently labeled sections were acquired for each hemisphere and were fused. All cortical 
A
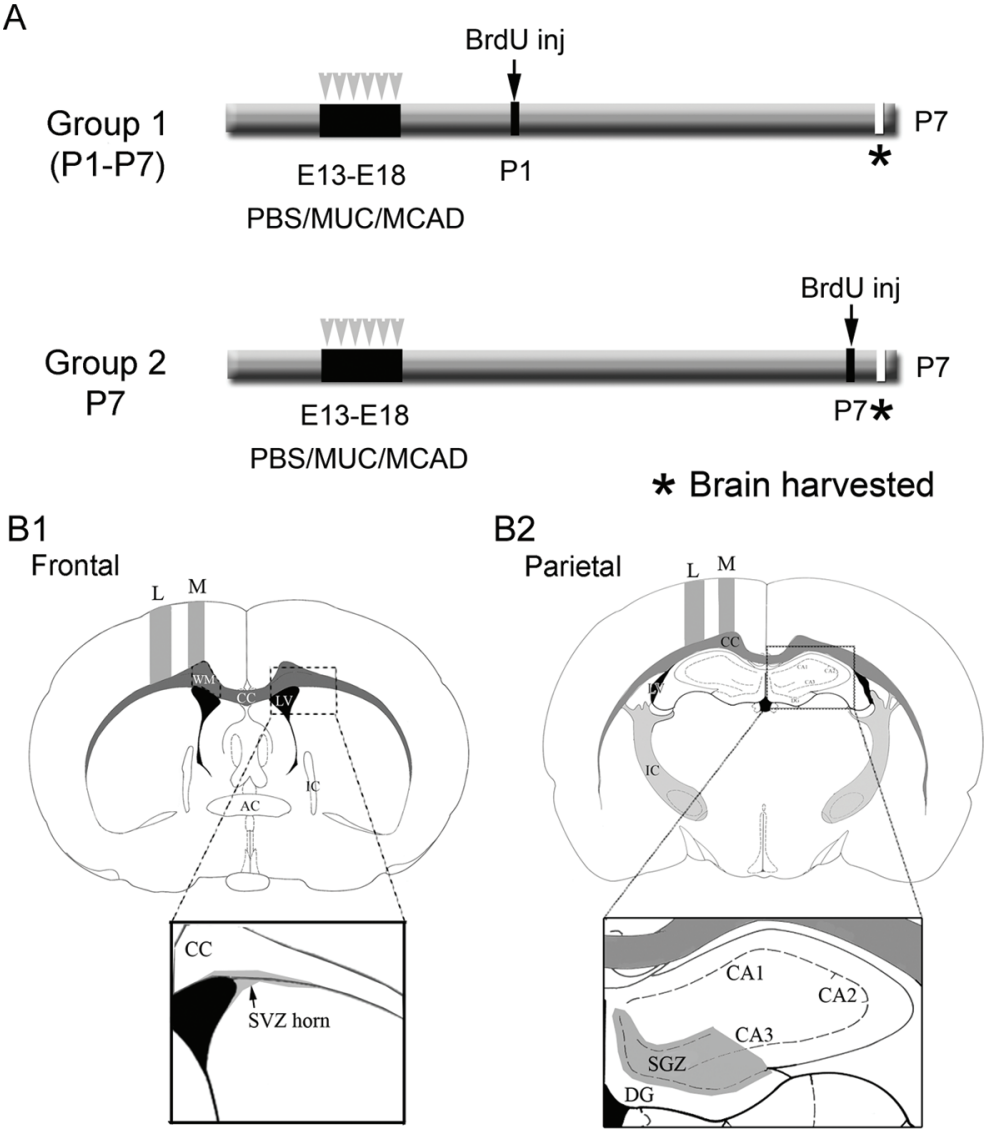

Figure 1. A. Schematics of experimental protocol. Group 1 represents the cell maturation and cell survival cohort and group 2, the proliferation profile for postnatal neurogenesis. B. Representation of ROls investigated to examine stem-cell niche proliferation and BrdU-positive cell distributions in neocortex and hippocampus. The sections represent mouse brain atlas coordinates: A. Frontal $\sim$ bregma $0.70-0.90 \mathrm{~mm}$ and B. Parietal $\sim$ bregma $-1.70-1.90 \mathrm{~mm}{ }^{12} \mathrm{Section}$ A represents the frontal coordinate for SVZ anterior horn analysis and section B represents the parietal coordinate for SGZ analysis (see magnified views). Abbreviations: L, lateral; M, medial; WM, white matter; CC, corpus callosum; LV, lateral ventricle; IC, internal capsule; DG, dentate gyrus; CA, Ammon's horn of hippocampus and respective subfields.

regions of interest (ROIs) are shown in Figure 1B. The more rostral area (B1) was designated as frontal and is at the level of the SVZ. The more caudal area (B2) was designated as parietal and is at the level of the SGZ. At each level, medial and lateral areas (rectangle $200 \mu \mathrm{m}$ width of cortical column for medial and $300 \mu \mathrm{m}$ for lateral cortex) were demarcated. BrdU-positive cell counts within these areas were reported asdensities (counts per ROI/area of ROI $\times 1000$ ). Cortical laminar counts were conducted by demarcating lamina using nuclear stain Hoechst. The differences in the BrdU-positive cell labeling between protocols used for groups 1 and 2 are shown in Suppl. Figure 1. BrdU-positive cells in the SGZ were quantified using Image $\mathrm{J}$ software (National Institutes of Health, Bethesda, MD, USA, http://rsb.info.nih.gov/ij/, 1997-2006). Single-channel green fluorescent images were converted into binary formats and intensity thresholds were kept constant for each fluorescent cell-type marker. These upper and lower limits of threshold intensity were determined for each stain such that background was satisfactorily eliminated. Counts of Iba- 1 positive cells and BrdU/Iba-1colabeled cells and Aldh1 L1-stained astrocytes were performed using an image analysis module in Axiovision and counts were conducted using markers and densities reported (counts per $\mathrm{ROI} /$ area of ROI $\times 1000$ ). Counts of NeuN stained neurons were performed using Stereo investigator (Micro Brightfield, Williston, VT, USA) using a $100 \times$ oil objective and optical fractionator for each cortical ROI. Counting frame width and height was $30 \mu \mathrm{m}$. Grid size for systematic random sampling was $200 \times 90 \mu \mathrm{m}$. Stereo investigator densities are reported for NeuN-positive cells.

Methods of analysis. All investigators performing quantitative analyses were blinded to animal identification and treatment assignments. Data were analyzed (Prism 4, Graph Pad Software, Inc, La Jolla, CA, USA) using one-way analysis of variance and post-hoc Newman-Keuls analyses. Means \pm SEM were reported and a probability below 0.05 was considered significant.

\section{Results}

No significant differences in cortical thickness in cortical ROIs at P7 with MCAD. Quantitation for total and laminar thickness was performed at the frontal and parietal 

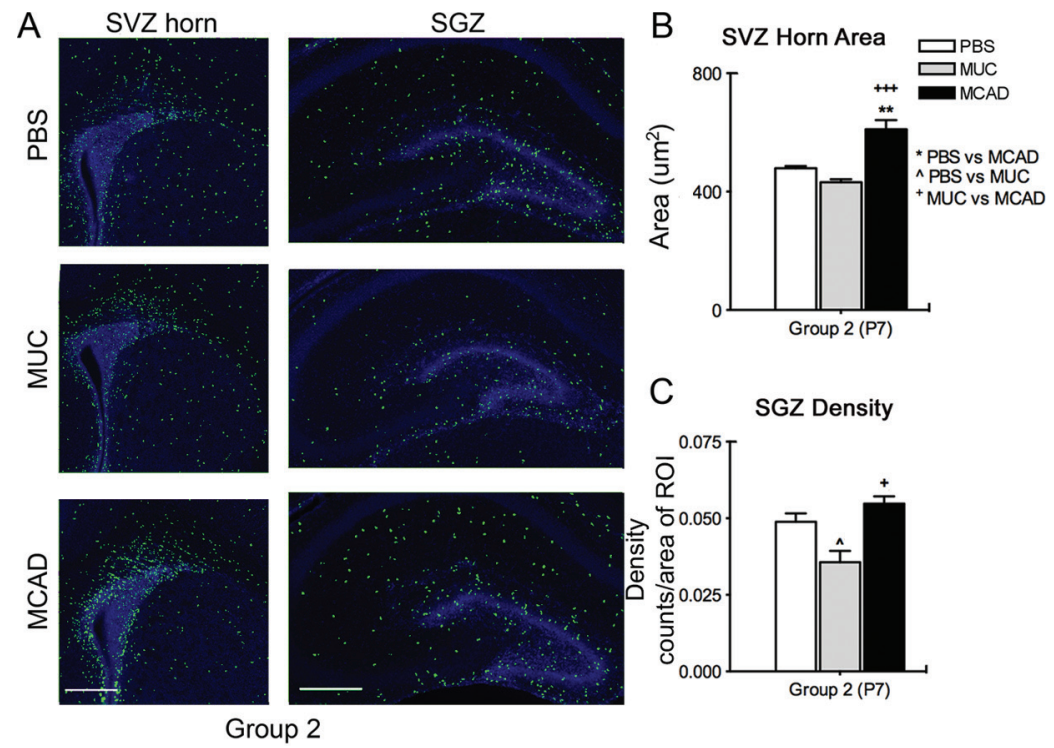

Density of BrdU Labeled Cells in Layers 2-4 of Cortex
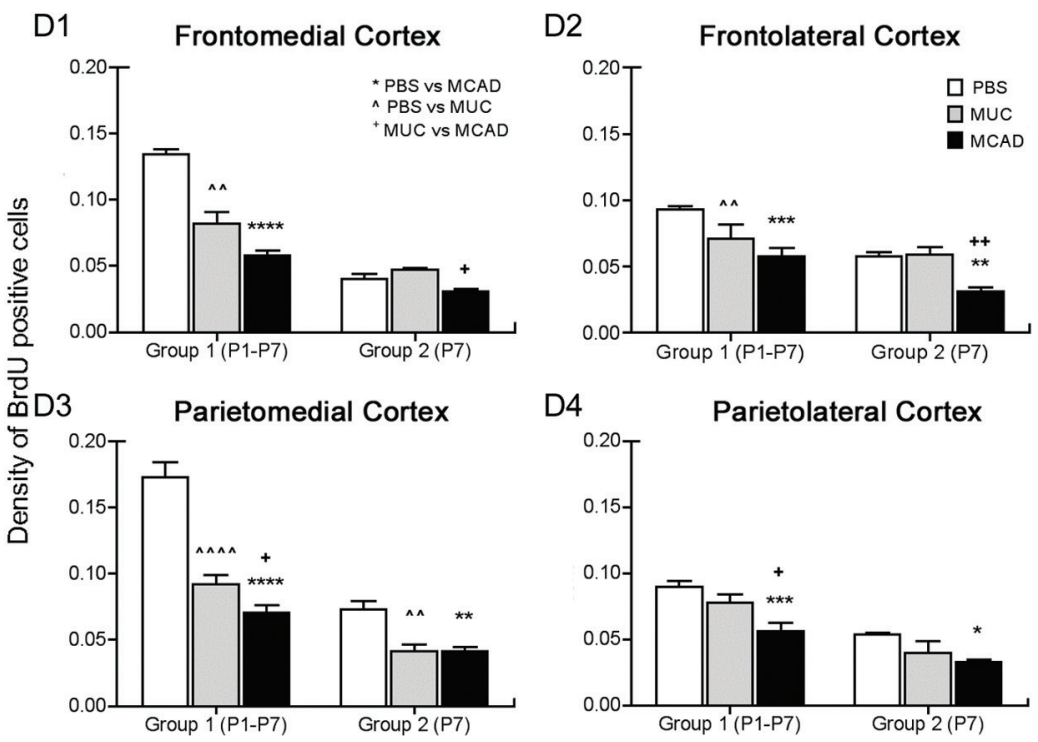

Figure 2. A, B, and C, MCAD-treated mice have increased BrdU-positive cell proliferation in neurogenic niches (group 2). A. Immunohistochemically labeled BrdU-positive nuclei (green) in representative sections from treatment groups show increased cell proliferation in both the SVZ anterior horn and DG that includes the SGZ born neurons. B. MCAD significantly increased neurogenic niche proliferative activity at P7 in sharp contrast to MUC. C. Image-J quantified counts of BrdU-positive cells densities indicate that MCAD significantly increased neurogenic niche proliferative activity at P7 again in sharp contrast to MUC ${ }^{*} P<0.05 ;{ }^{* *} P<0.01,{ }^{* * *} P<0.001$; scale bar $=200 \mu \mathrm{m} \mathrm{D}$. Decreased density of BrdU-positive cells in cortex (group 1 and 2 ). D1 and $\mathbf{2}$. Frontal cortex: a significant decrease in BrdU-positive cell densities in MCAD versus MUC treated groups detected in both the medial and lateral frontal cortex for group 2. D3 and 4. Parietal cortex: significant decreases in BrdU-positive cell densities in MCAD versus the MUC group in both the medial and lateral parietal lateral cortex in group 1. Although sample sizes were lower in group 2 the results complement group 1 data. ${ }^{*} P<0.05 ;{ }^{* \star} P<0.01 ;{ }^{* * *} P<0.001$; ${ }^{* \star * *} P<0.0001$.

coordinates illustrated in Figure 1B. In P7 pups, no significant differences in cortical thickness were observed among the treatment groupsat either the frontal or parietal ROIs examined (Suppl. Fig. 2; representative frontomedial cortex measurement data shown). All cortical lamina specific counts described below were conducted using Hoechst staining in the double-immunolabeled sections.

Effect of MCAD IgG on proliferation in the neurogenic niches of the SVZ and SGZ at P7. BrdU-labeled cells originating in the anterior horn of the SVZ and the SGZ were quantified in ROIs as illustrated in Figure 1B for group 2. Proliferating cells in SVZ were tightly packed and accurate quantitation by density was not possible since individual and stacked cells were not discernible at high magnification. In its place, we measured the area of the packed BrdU-positive cells in the SVZ horn (ie, using the green channel for BrdU-positive cells only; Fig. 2A), the expansion of which has been shown to represent increased proliferation in many rodent models of 
brain development, injury, and inflammation. ${ }^{14-16}$ SVZ areas (ie detected by borders of tightly packed BrdU-labeled cells) were significantly larger in the MCAD-IgG-treated group than in both the MUC IgG and PBS controls. In addition to the increased proliferation evident for MCAD treatment (Fig. 2B), a higher number of BrdU-labeled cells was also detected in the white-matter overlying the SVZ horn in the coronal sections.

In the SGZ, Image J quantified counts of BrdU-positive cell densities from the same brains are reported. The density of BrdU-labeled cells (ie, Image J counts/area) in the MCADIgG-treated group was significantly higher than in the MUC $\mathrm{IgG}$ group. Additionally, the MUC IgG group density was significantly lower than the PBS-treated group (Fig. 2C). Therefore MCAD exposure in utero resulted in a significant increase in BrdU-labeled cell proliferation at P7 compared to MUC IgG group both in the SVZ and SGZ when quantified using 2 different methods.

Significant laminar down regulation of BrdU-positive cell density in P7 neocortex. Laminar-specific cortical analysis showed significant PBSvs treatment decreases in BrdU-labeled cell density in the frontomedial and parietomedial cortices of the MCAD exposed pups that were not evident when all cortical layers were grouped together. Group 1 showed significantly decreased BrdU-positive cell density both in MUC-and MCAD-treated pups compared to PBS treatment (Fig. 2D). However, MCAD-treated pups had significantly lower densities compared to MUC in the parietal cortex and therefore was location-specific. Group 2 showed consistently lowered BrdU-positive cell proliferative density for MCAD treatment. Local cell proliferation with the 2-h protocol in MCAD-treated pups also was reduced significantly more than in MUC-treated pups in frontal cortical areas in group 2, but the effects of MUC were variable (Fig. 2D). Since laminar neuronal cortical migration is complete by P6 in mouse brains, the P7 cortically labeled BrdU-positive cells in group 2 may represent local glial cell populations that have recently been shown to continue to proliferate by symmetric cell division in the first few weeks after birth in rodents. ${ }^{17}$

Microglial densityin group $\mathbf{1}(\mathbf{n}=\mathbf{3 5})$. To assess the role of inflammation after MUC and MCAD exposure inutero, the density of Iba- 1 positive cells that developmentally enter the brain through systemic circulation ${ }^{18}$ was assessed in group 1 (ie, cell survival/maturation group; $\mathrm{n}=35$; see Suppl. Table 1). No significant increases in overall Iba-1 densities were noted in all ROIs examined except for SVZ, for which the MUC group showed significant increases in the density of Iba1-positive cells compared to PBS $(P<0.05)$ but not MCAD. Additionally, the percent of Iba-1-positive cells that co-labeled with BrdU were analyzed in fused $z$-stacks to quantitate BrdU-Iba-1 co-labeled cells in group 1. Iba1 and BrdU colocalization studies indicated that approximately $5-15 \%$ of the BrdU-labeled cells were microgliain all regions analyzed
(Suppl. Fig. 3). In SVZ, a significantly higher proportion of Iba-1-positive cells (20-25\% cells) co-labeled with $\mathrm{BrdU}$ in both MUC and MCAD groups compared with the PBS group (Suppl. Fig. 3E). The MUC group also showed a significantly higher proportion of Iba1- and BrdU-colabeled cells in the white-matter overlying the SVZ horn (Suppl. Fig. 3G; ROI marked over corpus callosum in Fig. 1B1). Therefore, both the MCAD and MUC treatment groups showed a higher density of postnatally born microglia when quantitated at P7, suggesting a xenograft effect or an increased reactivity of resident microglia to the $\mathrm{IgG}$.

Astrocytes and neuronal cortical density in group 1 $(\mathbf{n}=\mathbf{3 5})$. Densities of astrocytes (Suppl. Fig. 4A) in all ROIs examined (see Suppl. Table 2) showed a consistent but insignificant trend of decreased counts in layers 2-4 in the MCAD and MUC compared to PBS. Neuronal densities were not significantly different among the 3 treatment groups (Suppl. Table 2). The ratios of astrocyte to neuronal densities within the same cortex (Suppl. Fig. 4B) showed no significant differences among the treatment groups (data not shown).

\section{Discussion}

This study had the following significant findings: 1) MCAD exposure inutero led to significant alterations in postnatal cell proliferation at $\mathrm{P} 7$; 2) The postnatal effects of MCAD exposure on cell survival/local proliferation as detected by BrdU-labeled cell-densities in cortex were lower than MUC in frontal cortex and given recent reports ${ }^{17}$ of postnatal local proliferation of astrocytes in neocortex, the proliferative deficits in cortex in 2-h group at P7 may likely represent late onset local glial deficits. 3) Overall, microglial densities were not significantly higher in either the MCAD or MUC group; however, Iba-1 cells co-labeled with BrdU were detected at higher rates in the SVZ both after MUC and MCAD prenatal exposure and may represent a humanto-mouse xenograft effect or local inflammatory reactivity to the human $\mathrm{IgG}$.

The autistic brain changes during development and agespecific anatomical changes may result from age-specific molecular and circuit anomalies ${ }^{19}$ that require broader timepoints and profiles of investigation. This preliminary study was limited by the developmental ages and profiles covered; however, it reports novel MCAD specific postnatal anatomical effects on cell proliferation and highlights the importance of having additional human positive controls (ie, MUC in this study) when using mouse models. Although the proliferative neurogenic niche results in group 2 (ie, 2-h group) warrant repetition, similar trends in both the SGZ and SVZ zones and lack of large variability in the dataset are encouraging. The cell survival data in group 1 with a larger sample size showed fewer BrdU-positive cells in the cortex at P7, which is a significant finding; however, in this study, we cannot conclude whether this was as a result of cell death or failure of migration. The decrease in BrdU-positive cell 
densities in group 2 neocortices, however, indicates impaired local proliferation at $\mathrm{P} 7$.

Altered cell proliferation at $\mathrm{P7}$ and implications for the maturing brain. On P7, MCAD inutero exposure resulted in increased proliferation of cells in both the major neurogenic niches of the cerebrum. However, cells that were labeled on $\mathrm{P} 1$ resulted in lower cortical BrdU-positive cell densities at P7. We do not know the cause of this impairment or the long-term effects. However, the study lays down the ground work for future directed experimental protocols and reports novel findings, the most important being that MCAD IgG modulates postnatal cell proliferation in significantly different ways as compared to the positive control for human IgG (ie, MUC IgG) in the model. Previously reported findings of the age dependence of MCAD auto antibodies reactivity in rat brains, ${ }^{20}$ where patterns of reactivity were detected to prenatal (gestational day 18), but not on postnatal (day 8) or in adult rat brain proteins, make these new findings of significant postnatal alterations in cell proliferation from prenatal $\mathrm{IgG}$ exposure salient. The evidence that only gestational exposure dictates reactivity in fetal brain and that exposure results in long-term effects on brain development postnatally may be of importance in understanding the mechanisms underlying the pathophysiology. ${ }^{21,22}$

Increased microglial infiltration or local reactivity seen in SVZ with both the MCAD- and MUC-treated offspring highlight the importance of carefully considering the effects of human-to-mouse graft modulation of the systemic immune system when evaluating the role of inflammation in similar models. This study highlighted that MUC IgG also significantly altered many of the parameters evaluated for MCAD IgG when compared to PBS. The study shows that interpretation of MCAD data when compared to only PBS control alone may not be accurate without an internal control. This study also highlighted the potential effects specific to MCAD treatment in regions where MCAD and MUC data were significantly different from each other.

Astrocytes were often considered to be helper cells to neurons. However, recent advances have shown that several brain disorders are characterized by astrocytic pathologies that play a major role in the transmission of information between neurons in the brain. ${ }^{23-26}$ There is evidence that deficits in astrocyte markers in autism may underlie altered neurotransmission. ${ }^{27,28}$ Our data showed no significant decrease in astrocytic density at P7; however, the lower cortical BrdU-labeled cell densities reported here in the 2-h group may be local and non-neuronal in origin. Therefore, long-term assessment of astrocytic densities in the MCAD cortex is warranted and will help guide future experiments to examine their role.

\section{Conclusion}

Several behavioral changes have been documented in the pregnant dam mouse model injected with antibodies from MCAD, but the mechanisms underlying those alterations in the immature brains are not known. Other rodent studies have demonstrated that the transplacental transfer of antibodies is capable of producing central nervous system symptoms and brain pathology in exposed offspring. ${ }^{10,29-31}$ However, this study demonstrated the differential effects of MCAD vs MUC after inutero exposure on postnatal cell proliferation and survival in a pregnant dam model.

\section{Abbreviations}

BrdU, Bromodeoxyuridine; SVZ horn, anterior horn of subventricular zone; SGZ, sub-granular zone of dentate gyrus; MCAD, mothers of children with autistic disorder; MUC, mothers of unaffected children.

\section{Author Contributions}

Conceived and designed the experiments: SDK, BMF, AWZ, MEB, HSS. Analyzed the data: SDK, BMF, CMB, STK, MEB, AWZ. Wrote the first draft of the manuscript: SDK, $\mathrm{BMF}, \mathrm{CMB}$. Contributed to the writing of the manuscript: SDK, BMF, MEB, HSS. Agree with manuscript results and conclusions: SDK, BMF, CMB, STK, MEB, AWZ, HSS. Jointly developed the structure and arguments for the paper: SDK, HSS. Made critical revisions and approved final version: SDK, HSS, AWZ. All authors reviewed and approved of the final manuscript.

\section{DISCLOSURES AND ETHICS}

As a requirement of publication the authors have provided signed confirmation of their compliance with ethical and legal obligations including but not limited to compliance with ICMJE authorship and competing interests guidelines, that the article is neither under consideration for publication nor published elsewhere, of their compliance with legal and ethical guidelines concerning human and animal research participants (if applicable), and that permission has been obtained for reproduction of any copyrighted material. This article was subject to blind, independent, expert peer review. The reviewers reported no competing interests.

\section{Supplemental Data}

Supplemental figure 1. Representative profiles of BrdUpositive cell distribution detected with $\mathrm{P} 1$ and P7 BrdU injections to evaluate cell survival (group 1) and proliferation (group 2) in PBS control pups at P7. Scale bar $=200 \mu \mathrm{m}$.

Supplemental figure 2. Cortical thickness is not significantly altered at P7 in frontal or parietal cortices. Cresyl violet staining was used to discern cortical laminar thickness in ROIs. No significant differences were noted for total or layer specific cortical thickness among treatment groups in this study at P7. Scale bar $=100 \mu \mathrm{m}$.

Supplemental figure 3. Iba-1-positive cells that colabeled with BrdU in Group 1.6A-G. Trends for increased colocalization of P1 labeled BrdU-positive cells with microglial marker Iba-1 were detected in a few ROIs investigated and found to be significantly higher in the SVZ horn and WM overlying the horn in group 1 for both MCAD and MUC treatments. $6 \mathrm{H}$. Representative immunostaining image shows examples of BrdU-positive cells (BrdU- green), Iba-1 
positive microglia (red), and BrdU-positive cells that matured into microglia (yellow) in the cortex. ${ }^{*} P<0.05$; ${ }^{* *} P<0.01$.

Supplemental figure 4. Newly discovered astrocyte specific marker ALDHL1 to quantitate cortical astrocytes. A. Uniform astrocyte label in white and gray matter allows for improved gray matter assessment. A'. Magnified view of gray matter label. B. Colabeling with $\mathrm{NeuN}$ allowed for evaluating astrocyte/neuronal density ratios within neocortex of same coronal sections.

Supplemental table 1. Density of Iba1 stained microglial cells in Group 1.

Supplemental table 2. Density of Aldh1L1 stained astrocytes and NeuN stained neurons in Group 1.

\section{REFERENCES}

1. APA Washington DC. Diagnostic and Statistical Manual of Mental Disorders, Text Revision. 4th ed. Washington, DC: American Psychiatric Association. 2000

2. Zimmerman AW. Autism: Current Theories and Evidence. New York, NY: Humana Press; 2008.

3. Braunschweig D, Ashwood P, Krakowiak P, et al. Autism: maternally derived antibodies specific for fetal brain proteins. Neurotoxicology. 2008;29(2):226-231.

4. Braunschweig D, Duncanson P, Boyce R, et al. Behavioral correlates of maternal antibody status among children with autism. J Autism Dev Disord. 2011; 42(7):1435-1445.

5. Fourgeaud L, Boulanger LM. Role of immune molecules in the establishment and plasticity of glutamatergic synapses. Eur J Neurosci. 2010;32(2):207-217.

6. Goines PE, Croen LA, Braunschweig D, et al. Increased midgestational IFNgamma, IL-4 and IL-5 in women bearing a child with autism: a case-control study. Mol Autism. 2011;2:13.

7. Braunschweig D, Van de Water J. Maternal autoantibodies in autism. Arch Neurol. 2012;69(6):693-699.

8. Braunschweig D, Ashwood P, Krakowiak P, et al. Autism: maternally derived antibodies specific for fetal brain proteins. Neurotoxicology. 2008;29(2):226-231.

9. Dantzer R, Kelley KW. Autistic children: a neuroimmune perspective. Brain Behav Immun. 2008;22(6):804-805.

10. Singer HS, Morris C, Gause C, Pollard M, Zimmerman AW, Pletnikov M. Prenatal exposure to antibodies from mothers of children with autism produces neurobehavioral alterations: a pregnant dam mouse model. J Neuroimmunol. 2009; 211(1-2):39-48

11. Martin LA, Ashwood P, Braunschweig D, Cabanlit M, Van de Water J, Amaral DG. Stereotypies and hyperactivity in rhesus monkeys exposed to IgG from mothers of children with autism. Brain Behav Immun. 2008;22(6):806-816.
12. Paxinos G, Franklin KBJ. The Mouse Brain in Stereotaxic Coordinates: Compact Second Edition. Waltham, MA: Elsevier Science \& Technology Books; 2003.

13. Cahoy JD, Emery B, Kaushal A, et al. A transcriptome database for astrocytes, neurons, and oligodendrocytes: a new resource for understanding brain development and function. J Neurosci. 2008;28(1):264-278.

14. Kadam SD, Mulholland JD, McDonald JW, Comi AM. Poststroke subgranular and rostral subventricular zone proliferation in a mouse model of neonatal stroke. JNeurosci Res. 2009;87(12):2653-2666.

15. Brazel CY, Romanko MJ, Rothstein RP, Levison SW. Roles of the mammalian subventricular zone in brain development. Prog Neurobiol. 2003;69(1):49-69.

16. Romanko MJ, Rola R, Fike JR, et al. Roles of the mammalian subventricular zone in cell replacement after brain injury. Prog Neurobiol. 2004;74(2):77-99.

17. Ge WP, Miyawaki A, Gage FH, Jan YN, Jan LY. Local generation of glia is a major astrocyte source in postnatal cortex. Nature. 2012;484(7394):376-380.

18. Ginhoux F, Lim S, Hoeffel G, Low D, Huber T. Origin and differentiation of microglia. Front Cell Neurosci. 2013;7:45.

19. Courchesne E, Campbell K, Solso S. Brain growth across the life span in autism: age-specific changes in anatomical pathology. Brain Res. 2011;1380:138-145.

20. Zimmerman AW, Connors SL, Matteson KJ, et al. Maternal antibrain antibodies in autism. Brain Behav Immun. 2007;21(3):351-357.

21. Soumiya H, Fukumitsu H, Furukawa S. Prenatal immune challenge compromises development of upper-layer but not deeper-layer neurons of the mouse cerebral cortex. J Neurosci Res. 2011;89(9):1342-1350.

22. Soumiya H, Fukumitsu H, Furukawa S. Prenatal immune challenge compromises the normal course of neurogenesis during development of the mouse cerebral cortex. J Neurosci Res. 2011;89(10):1575-1585.

23. Dalton $\mathrm{P}$, Deacon R, Blamire A, et al. Maternal neuronal antibodies associated with autism and a language disorder. Ann Neurol. 2003;53(4):533-537.

24. Vincent A, Dalton P, Clover L, Palace J, Lang B. Antibodies to neuronal targets in neurological and psychiatric diseases. Ann NY Acad Sci. 2003;992:48-55.

25. Diamond B, Bloom O, Al AY, Kowal C, Huerta PT, Volpe BT. Moving towards a cure: blocking pathogenic antibodies in systemic lupus erythematosus. J Intern Med. 2011;269(1):36-44.

26. Maezawa I, Swanberg S, Harvey D, LaSalle JM, Jin LW. Rett syndrome astrocytes are abnormal and spread $\mathrm{MeCP} 2$ deficiency through gap junctions. $J \mathrm{Neu}-$ rosci. 2009;29(16):5051-5061.

27. Nedergaard M, Ransom B, Goldman SA. New roles for astrocytes: redefining the functional architecture of the brain. Trends Neurosci. 2003;26(10):523-530

28. Verkhratsky A, Sofroniew MV, Messing A, et al. Neurological diseases as primary gliopathies: a reassessment of neurocentrism. ASN Neuro. 2012;4(3).

29. Dalton $P$, Deacon $R$, Blamire A, et al. Maternal neuronal antibodies associated with autism and a language disorder. Ann Neurol. 2003;53(4):533-537.

30. Vincent A, Dalton P, Clover L, Palace J, Lang B. Antibodies to neuronal targets in neurological and psychiatric diseases. Ann NY Acad Sci. 2003;992:48-55.

31. Diamond B, Bloom O, A1 AY, Kowal C, Huerta PT, Volpe BT. Moving towards a cure: blocking pathogenic antibodies in systemic lupus erythematosus. J Intern Med. 2011;269(1):36-44. 